Effects of Damage on Long-term Displacement Data of Woven Fabric Webbings Under Constant Load for Inflatable Structures

Winfred S. Kenner NASA LaRC

Structural Mechanics and Concepts Branch AIAA SciTech 2016, January 3-8, 2016 San Diego, California 
- Background

- Inflatable Structures

- Problem Statement

- Approach

- Hardware and Test Facility

- Test Results

- Summary 


\section{Inflatable Structures}

Research Materials:

Kevlar Webbing Vectran Webbing

Typical Roll of Webbing Materialo (Flexible Structural Restraint Layer)

\section{Typical Inflatable Structures}

$\sqrt{5}$

- Small

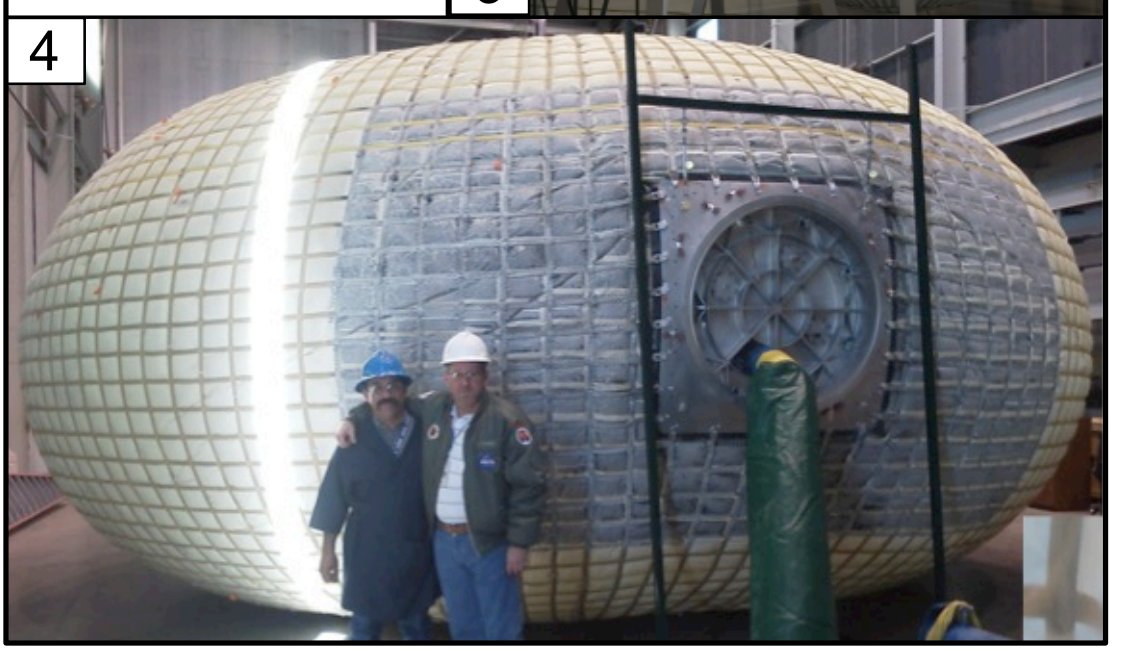
Storage Volume - Fast Build

2

- Lightweight

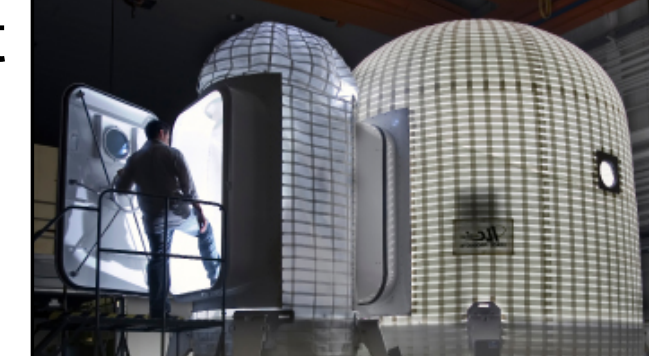
$3(4)=$ 
- Goal: Conduct an experimental creep study to identify effects of damage on webbing performance over extended time period

- Causation: Damage due to deployment and/or service

- Definition: Damage is defined as vertical incisions of varying length and number

- Analytical analysis: Inaccurate for nonlinear materials over time

- Time period: Study was conducted over an 18-month time period
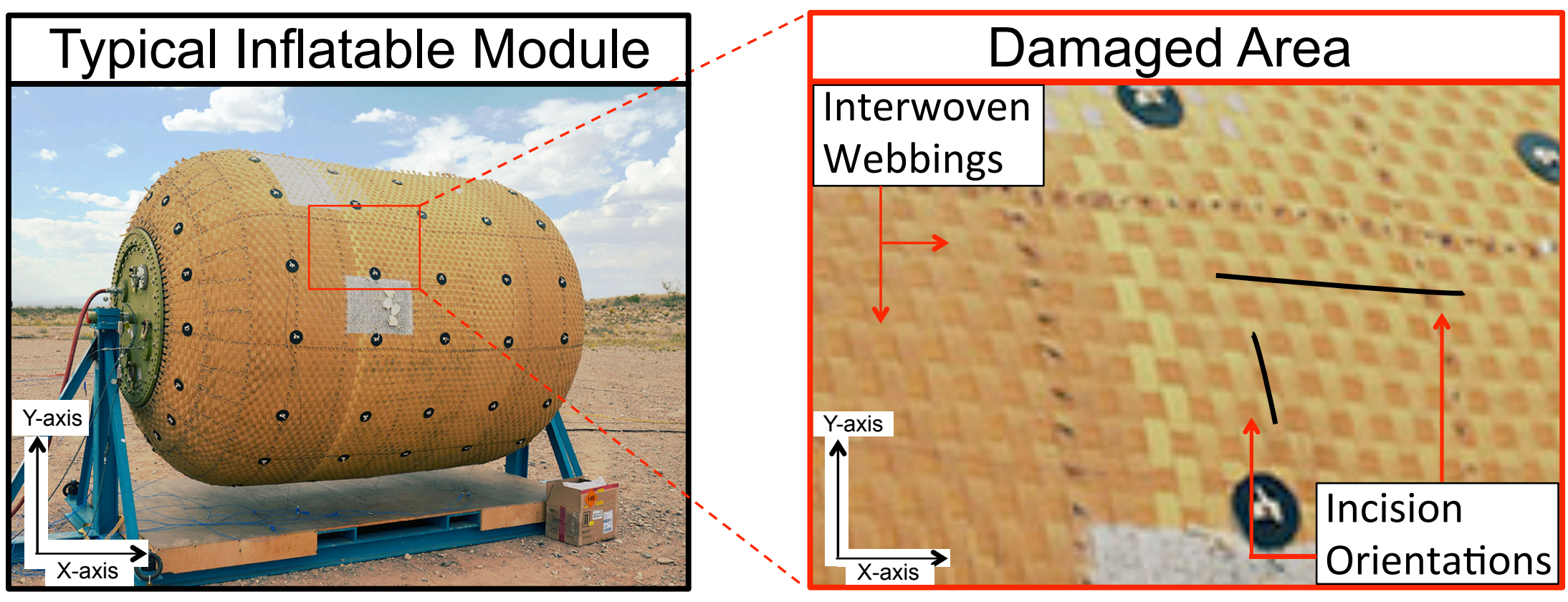
Goal: Generate long-term displacement data from webbings under load
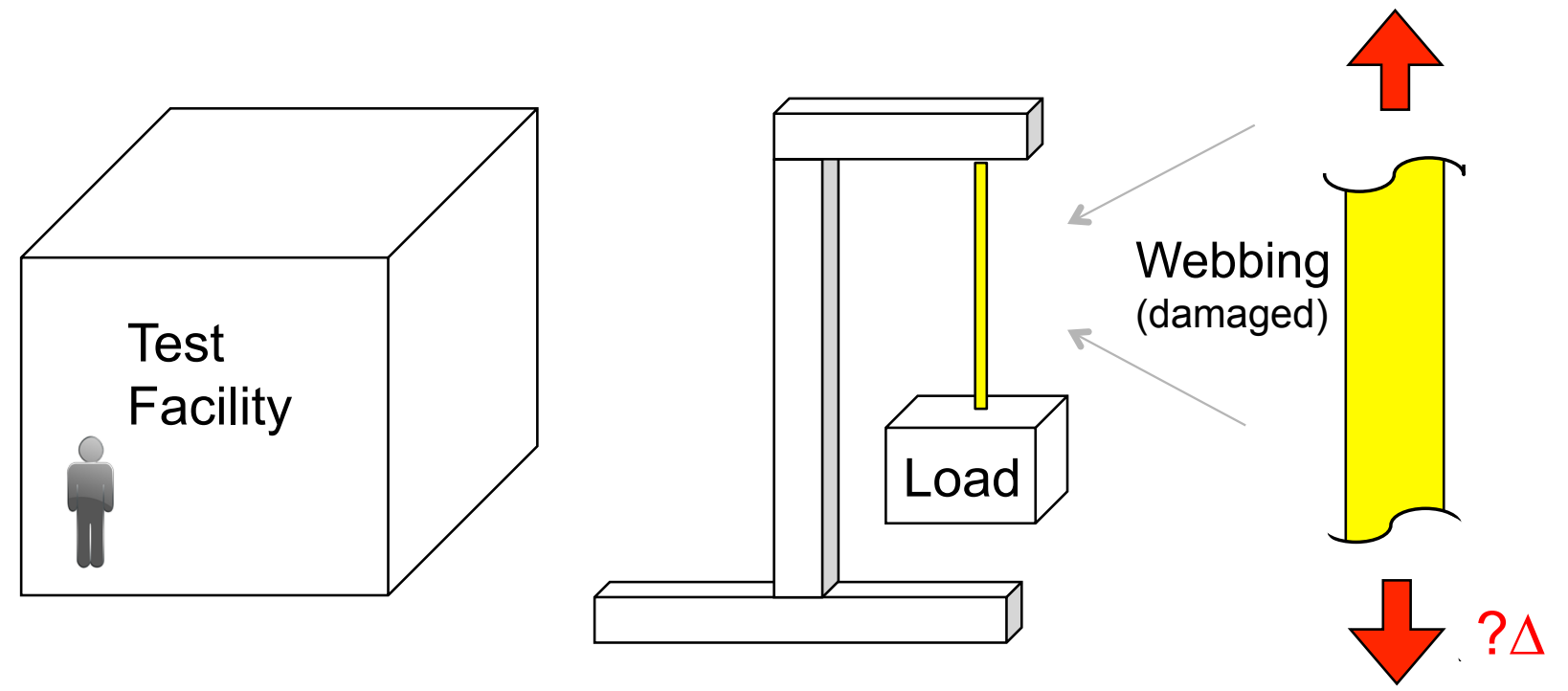

Computer system

\section{Test Fad}

- Overhead crane

Test Hardware

- High load capacity

- Quick assembly
Displacement Measuring

Device

- Accurate

- Continuous measurements

\section{Data} Acquisition

- Variable rate

- Continuous recordings over multiple years 


\section{Creep Test Facility}

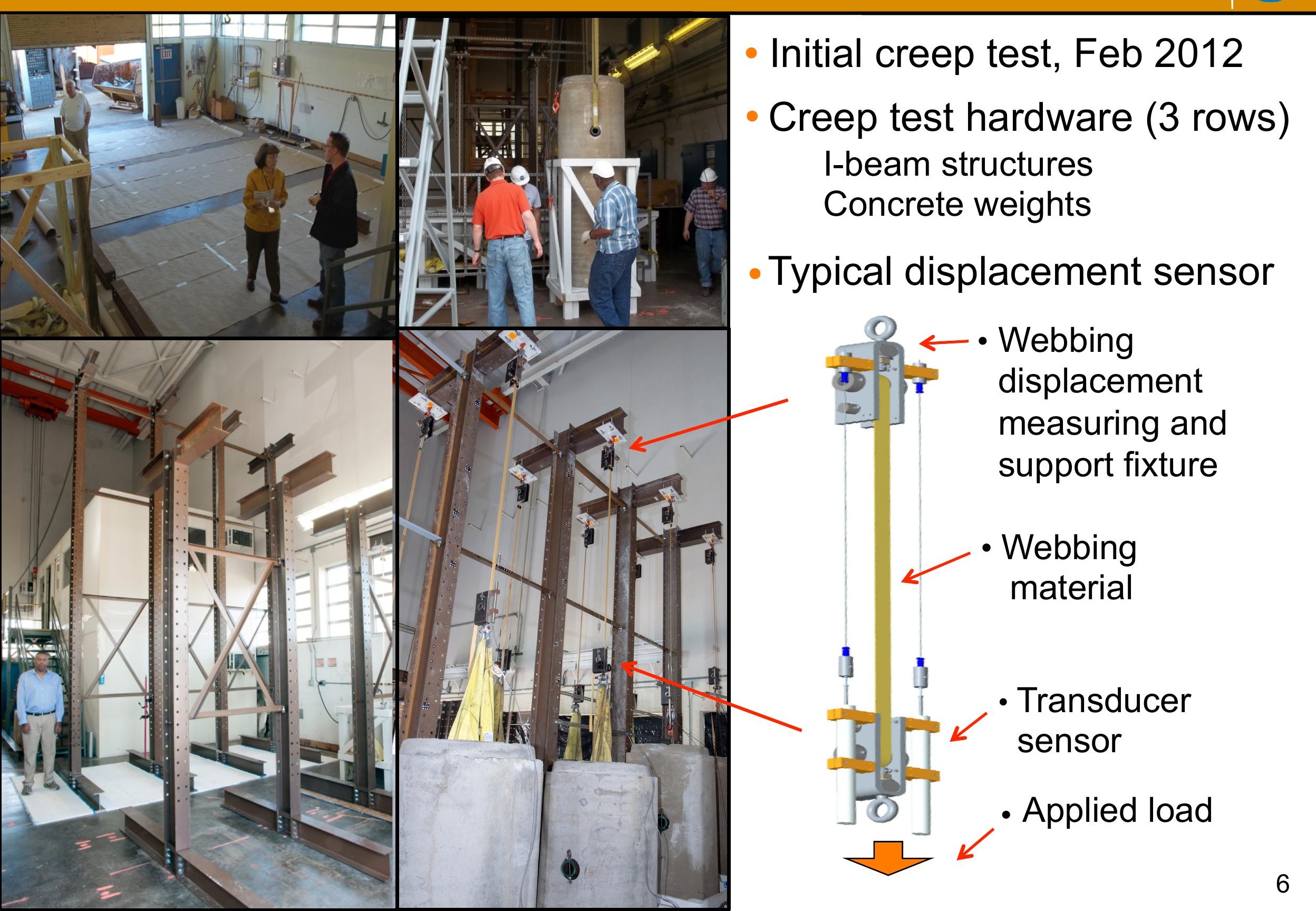


Magnified Webbing Images
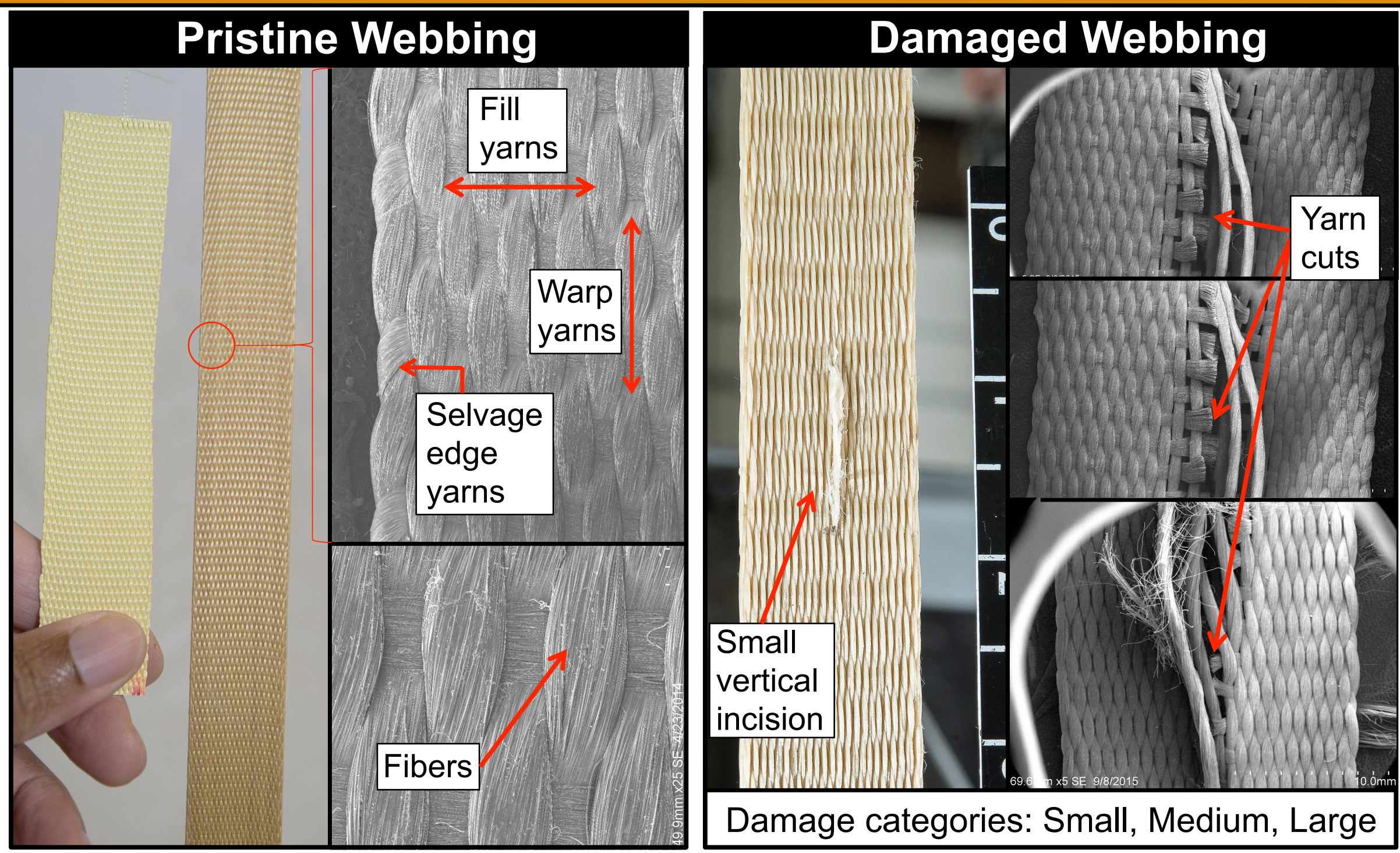

- Eleven test groups defined by test material, applied load, and damage - Horizontal incision: Known to have an adverse effect on strength proportional to length 


\section{Pristine Webbings}

\begin{tabular}{|c|l|c|c|c|c|}
\hline $\begin{array}{c}\text { Group } \\
\#\end{array}$ & $\begin{array}{l}\text { Webbing } \\
\text { materials }\end{array}$ & $\begin{array}{c}\text { Webbing } \\
\#\end{array}$ & $\begin{array}{c}\text { UTS } \\
(\%)\end{array}$ & $\begin{array}{c}\text { Webbing } \\
\text { length }\end{array}$ & $\begin{array}{c}\text { Length } \\
\text { of test } \\
\text { (months) }\end{array}$ \\
\hline 1 & $6 \mathrm{~K}$ & 1 & 20 & 144 & 34 \\
& Kevlar & 2 & 20 & 144 & 34 \\
\hline 2 & $6 \mathrm{~K}$ & 1 & 20 & 60 & 3 \\
& Kevlar & 2 & 20 & 60 & 3 \\
\hline 3 & $6 \mathrm{~K}$ & 1 & 43 & 68 & 39 \\
& Kevlar & 2 & 43 & 84 & 39 \\
& & 3 & 50 & 82 & 35 \\
& & 4 & 50 & 80 & 35 \\
\hline 4 & $6 \mathrm{~K}$ & 1 & 50 & 80 & 34 \\
& Vectran & 2 & 50 & 80 & 34 \\
& & 3 & 50 & 80 & 34 \\
\hline 5 & $12.5 \mathrm{~K}$ & 1 & 29 & 116 & 29 \\
& Vectran & 2 & 29 & 116 & 29 \\
& & 3 & 29 & 116 & 22 \\
\hline 6 & $6 K$ & 1 & 25 & 66 & 44 \\
& Vectran & 2 & 25 & 66 & 44 \\
& & 3 & 25 & 66 & 44 \\
& & 4 & 25 & 66 & 44 \\
& & 5 & 20 & 66 & 44 \\
\hline
\end{tabular}

\section{Damaged Webbings}

\begin{tabular}{|c|l|c|c|c|c|c|}
\hline $\begin{array}{c}\text { Group } \\
\#\end{array}$ & $\begin{array}{c}\text { Webbing } \\
\text { materials }\end{array}$ & $\begin{array}{c}\text { Webbing } \\
\#\end{array}$ & $\begin{array}{c}\text { UTS } \\
(\%)\end{array}$ & $\begin{array}{c}\text { Webbing } \\
\text { length }\end{array}$ & $\begin{array}{c}\text { Length } \\
\text { of test } \\
\text { (months) }\end{array}$ & $\begin{array}{c}\text { Incision } \\
\text { length } \\
\text { (inches) }\end{array}$ \\
\hline 7 & $6 K$ & 1 & 20 & 60 & 18 & 5 \\
& Kevlar & 2 & 20 & 60 & 16 & 5 \\
\hline \multirow{2}{*}{8} & $6 K$ & 1 & 6.7 & 60 & 14 & 1 \\
& Kevlar & 2 & 6.7 & 60 & 14 & 1 \\
\hline 9 & $6 K$ & 1 & 20 & 62 & 3 & 5 \\
& Kevlar & 2 & 20 & 62 & 3 & 5 \\
\hline 10 & $6 K$ & 1 & 20 & 62 & 3 & 5 \\
& Vectran & 2 & 20 & 60 & 18 & 5 (multiple) \\
& & 3 & 20 & 60 & 18 & 5 (multiple) \\
\hline 11 & $12.5 K$ & 1 & 29 & 48 & 18 & 1 \\
& Vectran & 2 & 29 & 41 & 18 & 1 \\
& & 3 & 29 & 43 & 18 & 1 \\
\hline
\end{tabular}

*Ultimate tensile strength (UTS) 


\section{Environmental Influences on Displacement (nses)}

\section{Displacement Data}

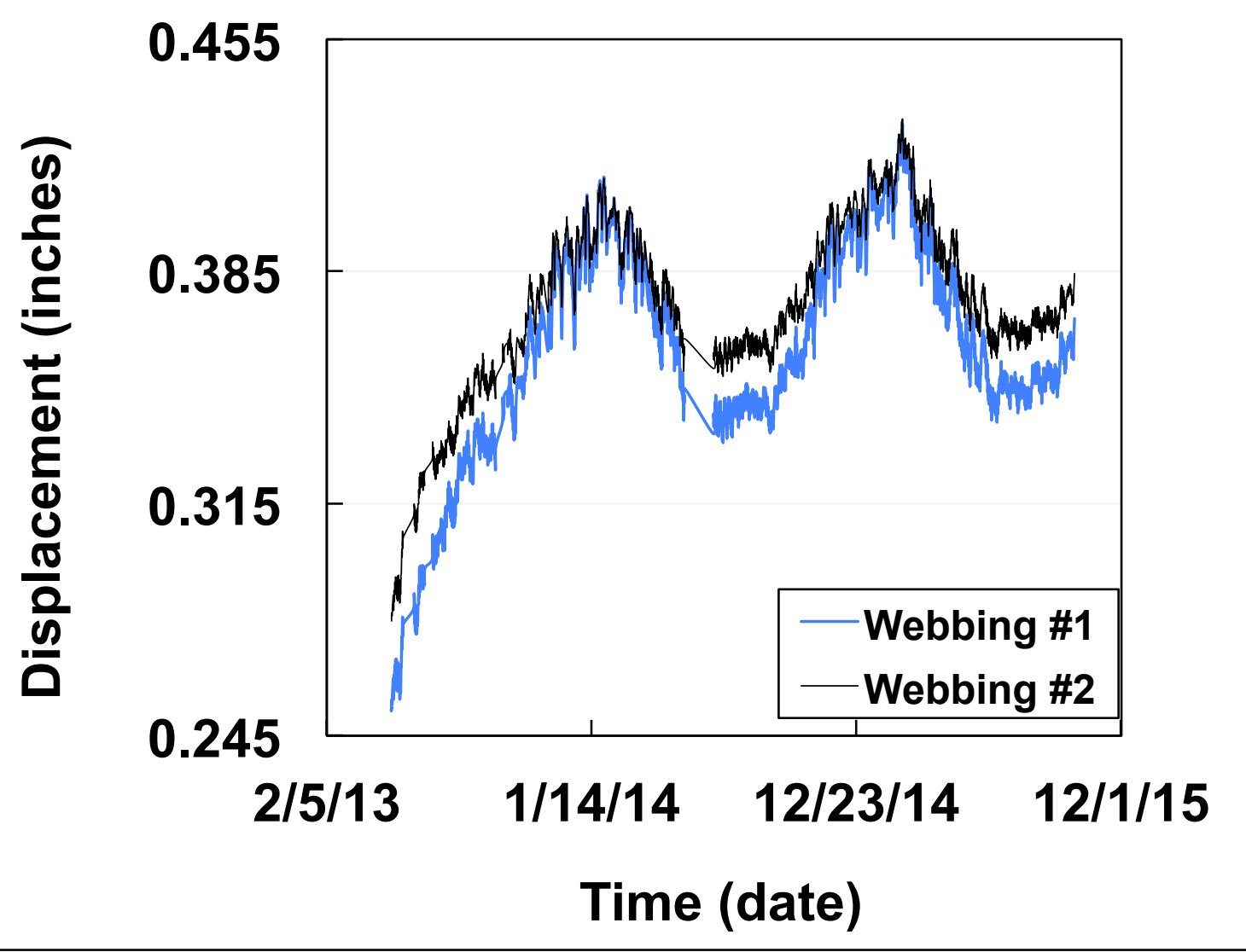

Temperature Data

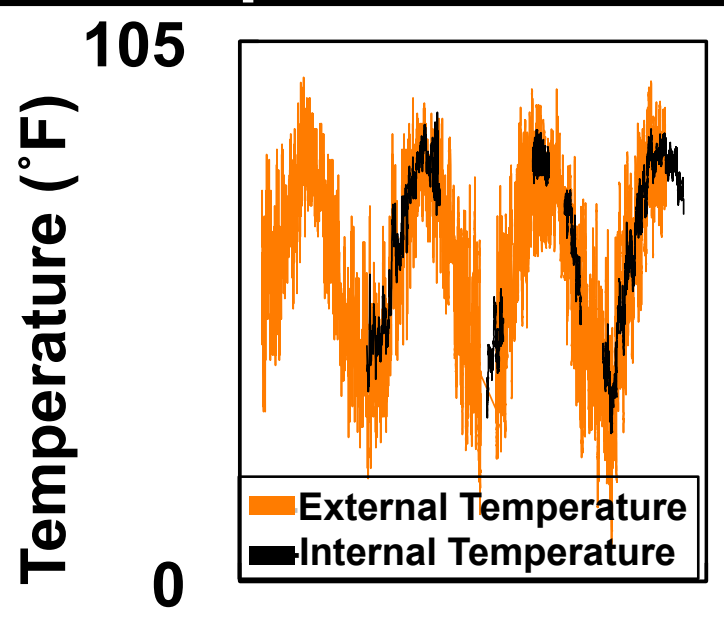

$12 / 23 / 11$

$12 / 12 / 15$

Time (date)

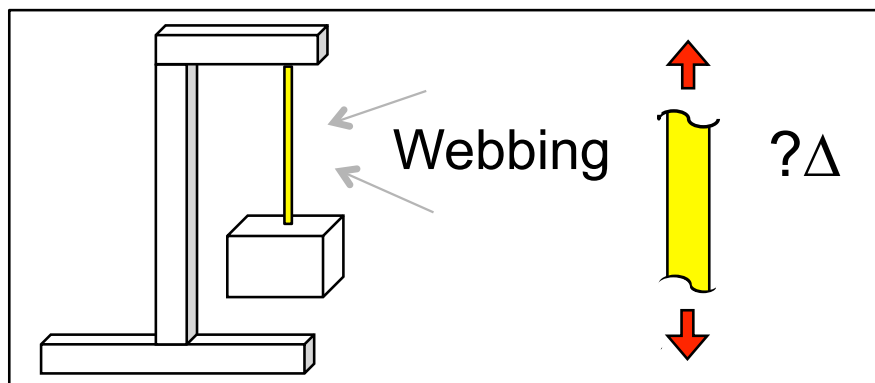

- Daily, seasonal, and yearly temperature and humidity effects influence the creep displacement curve -Webbings exhibits negative coefficient of thermal expansion (CTE) 


\section{Characteristics of Displacement Curve}

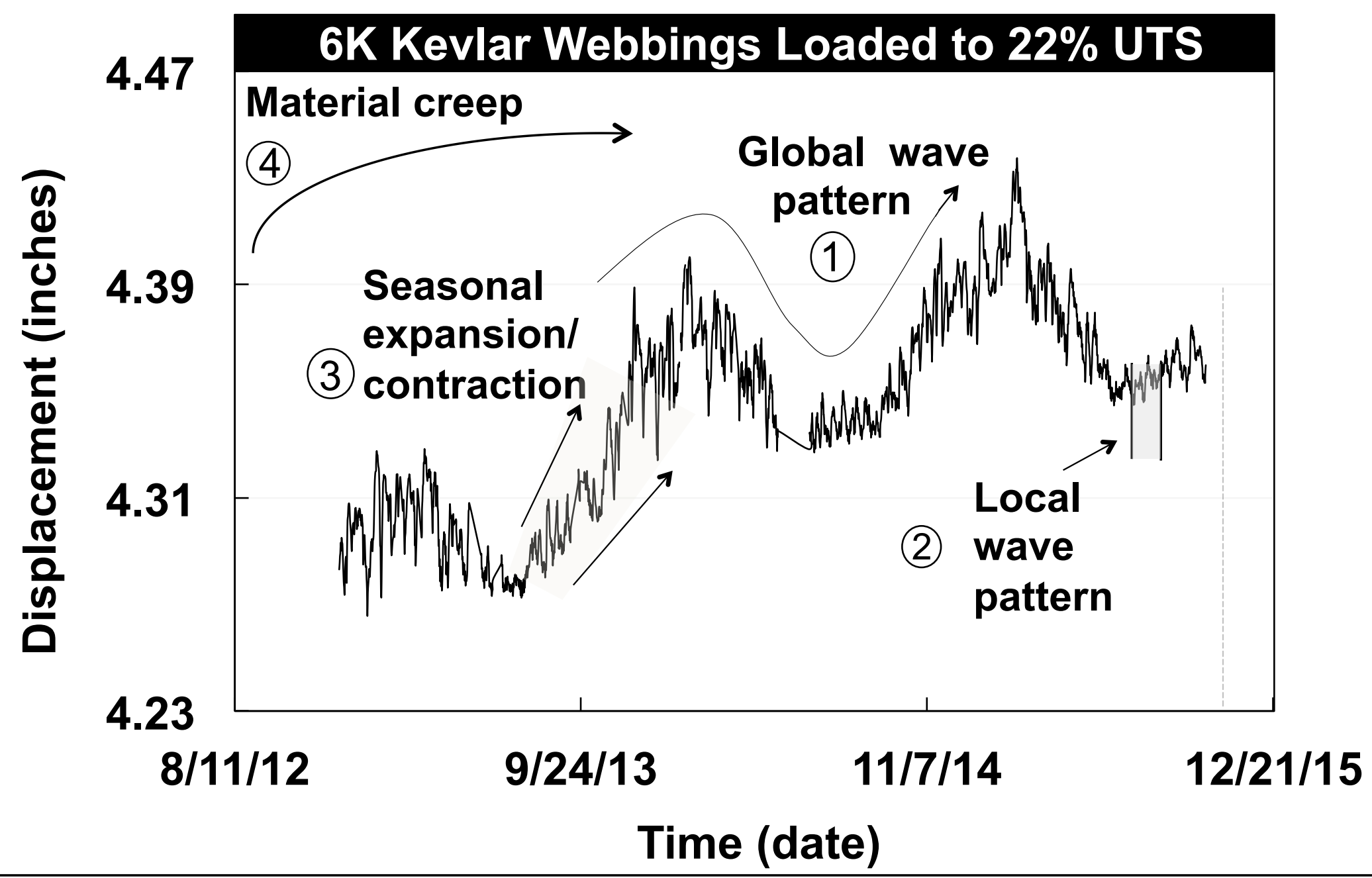

- 3-year time period

- Four characteristic curve patterns

- Failure experienced above $50 \%$ UTS

Pristine Webbing 


\section{Effects of Damage}

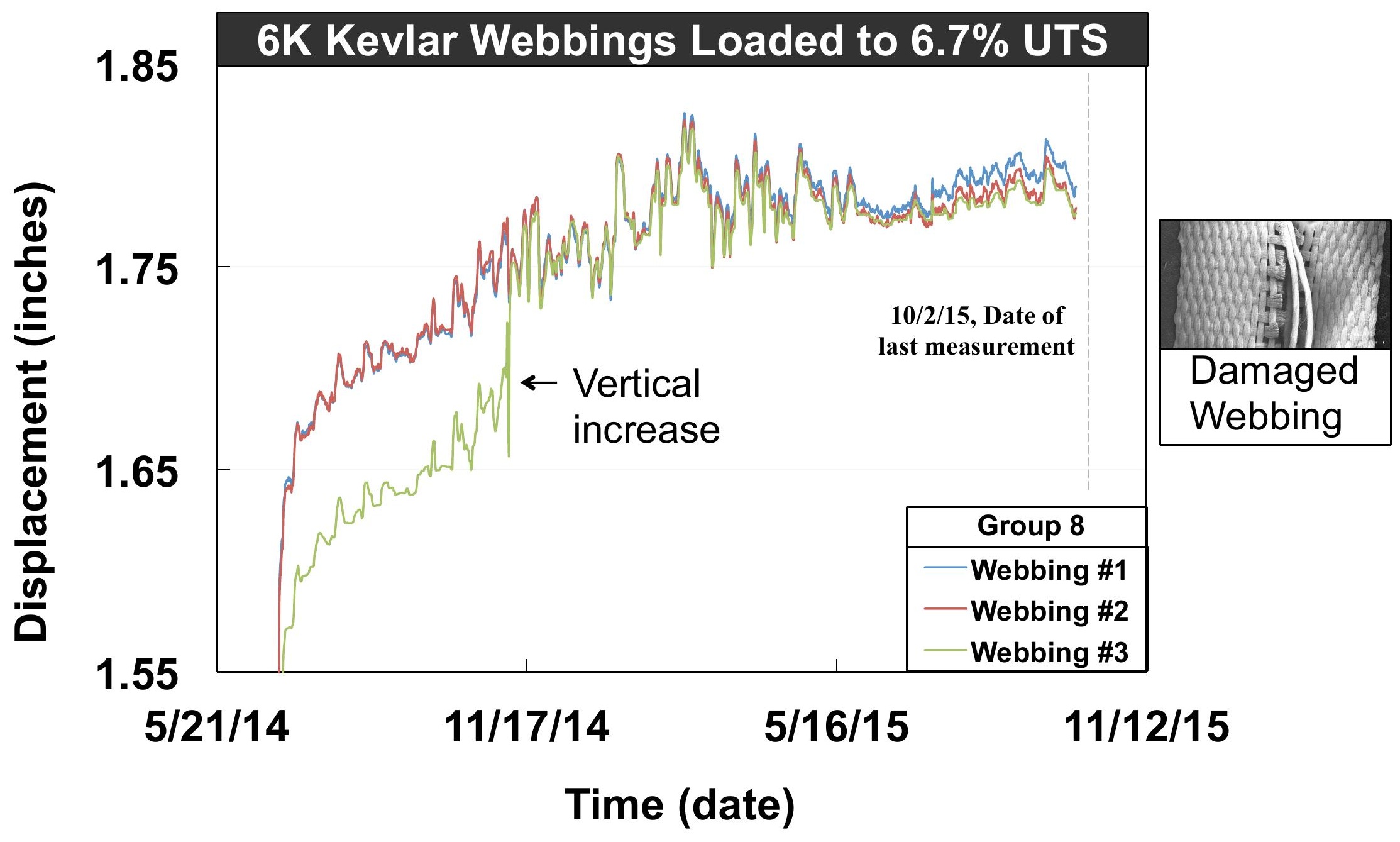

- Damage introduces vertical spikes into local wave pattern - Damage doesn't effect strength over time 


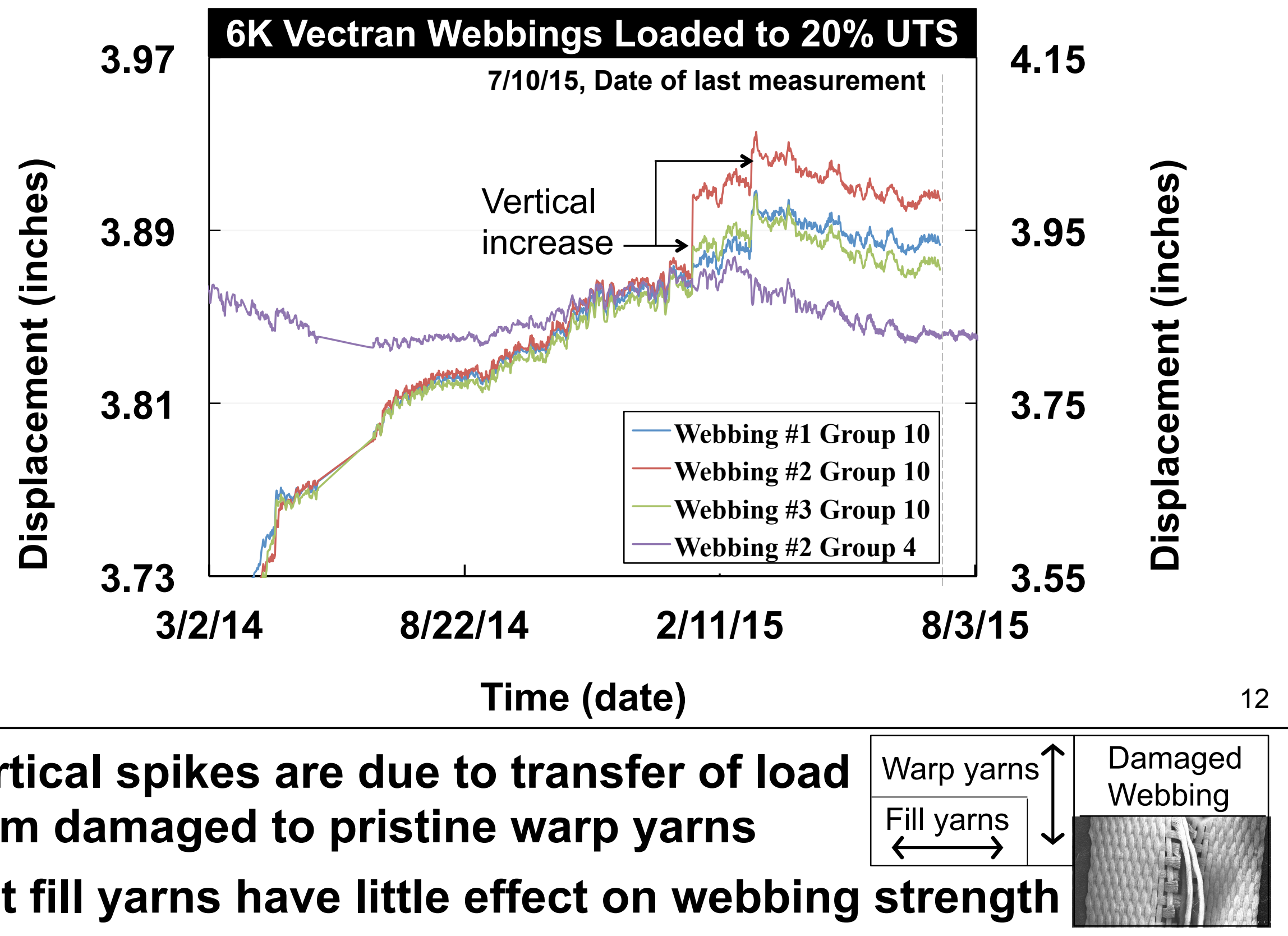

\begin{tabular}{l} 
- Vertical spikes are due to transfer of load $\left.\begin{array}{l}\text { Warp yarns } \uparrow \\
\text { from damaged to pristine warp yarns } \\
\text { - Cut fill yarns have little effect on webbing strength }\end{array}\right] \mid \begin{array}{l}\text { Damaged } \\
\text { Webbing }\end{array}$ \\
\hline
\end{tabular}




\section{Effects of Damage}

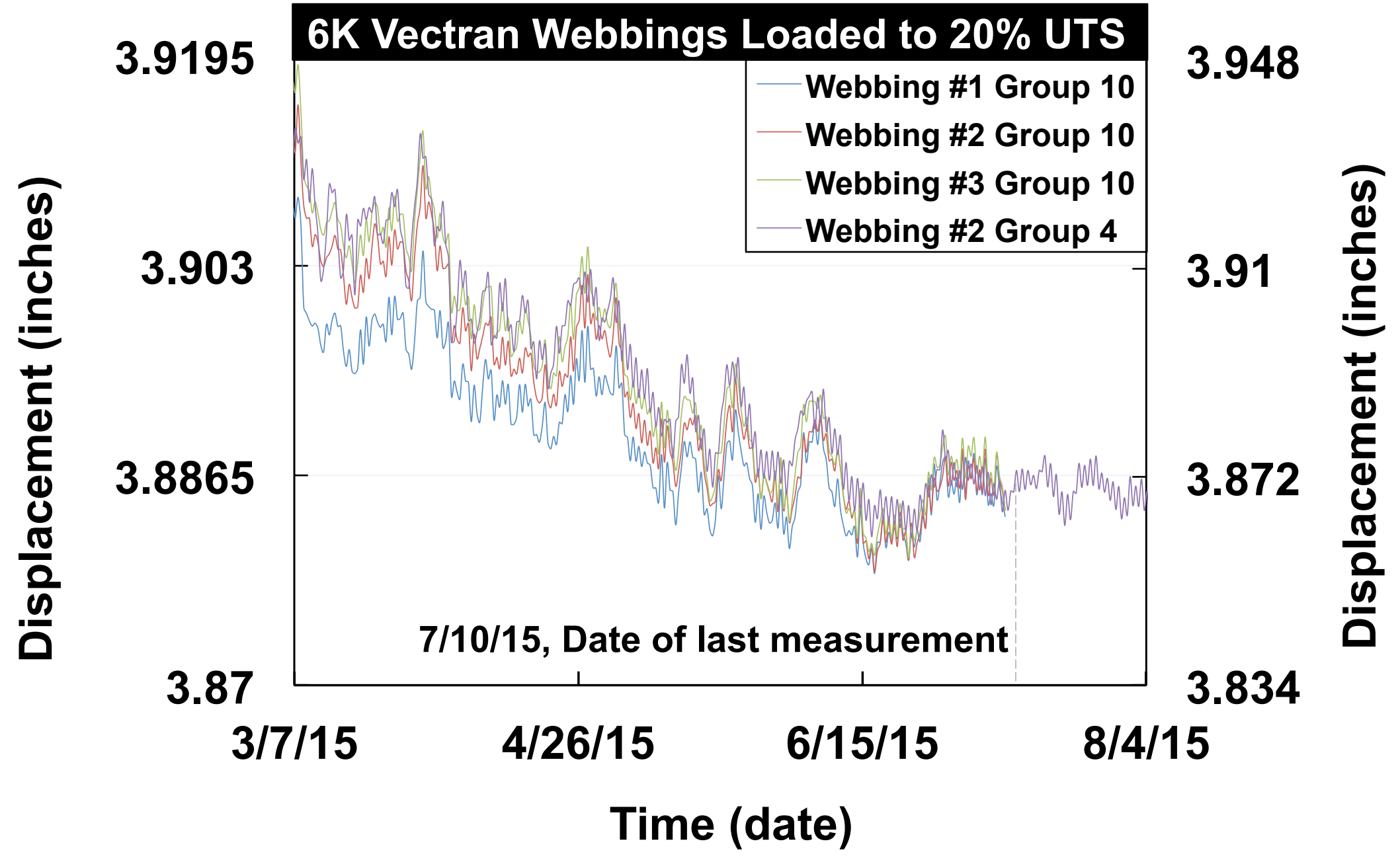

- Magnified view of damaged webbing wave patterns relative to baseline pattern indicates insignificant effect of damage 


\section{Effects of Rotation}

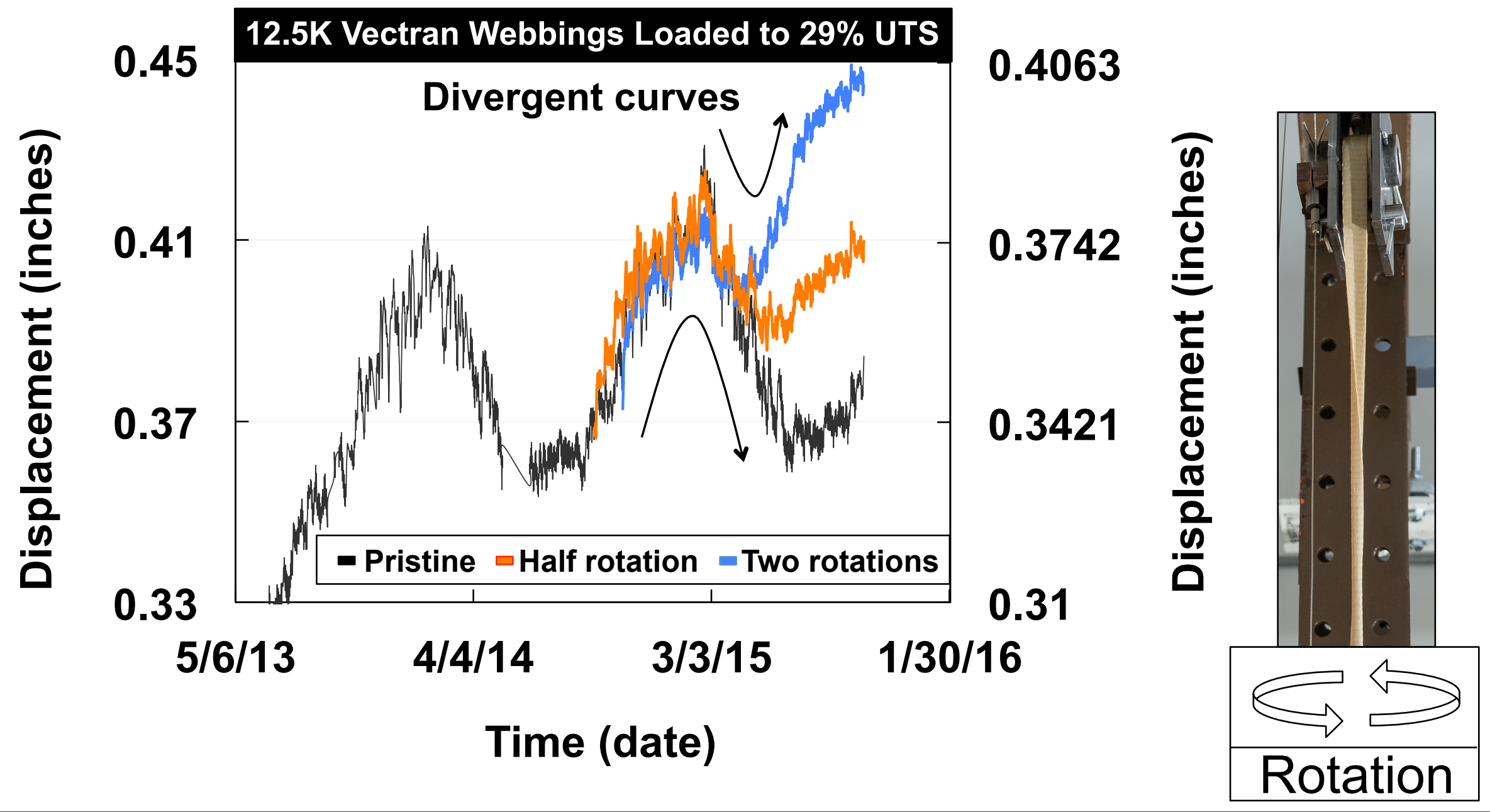

- Rotation introduces a divergent global wave pattern

- Rotation has a significant effect on strength over time 


\section{Running Sum Calculation}

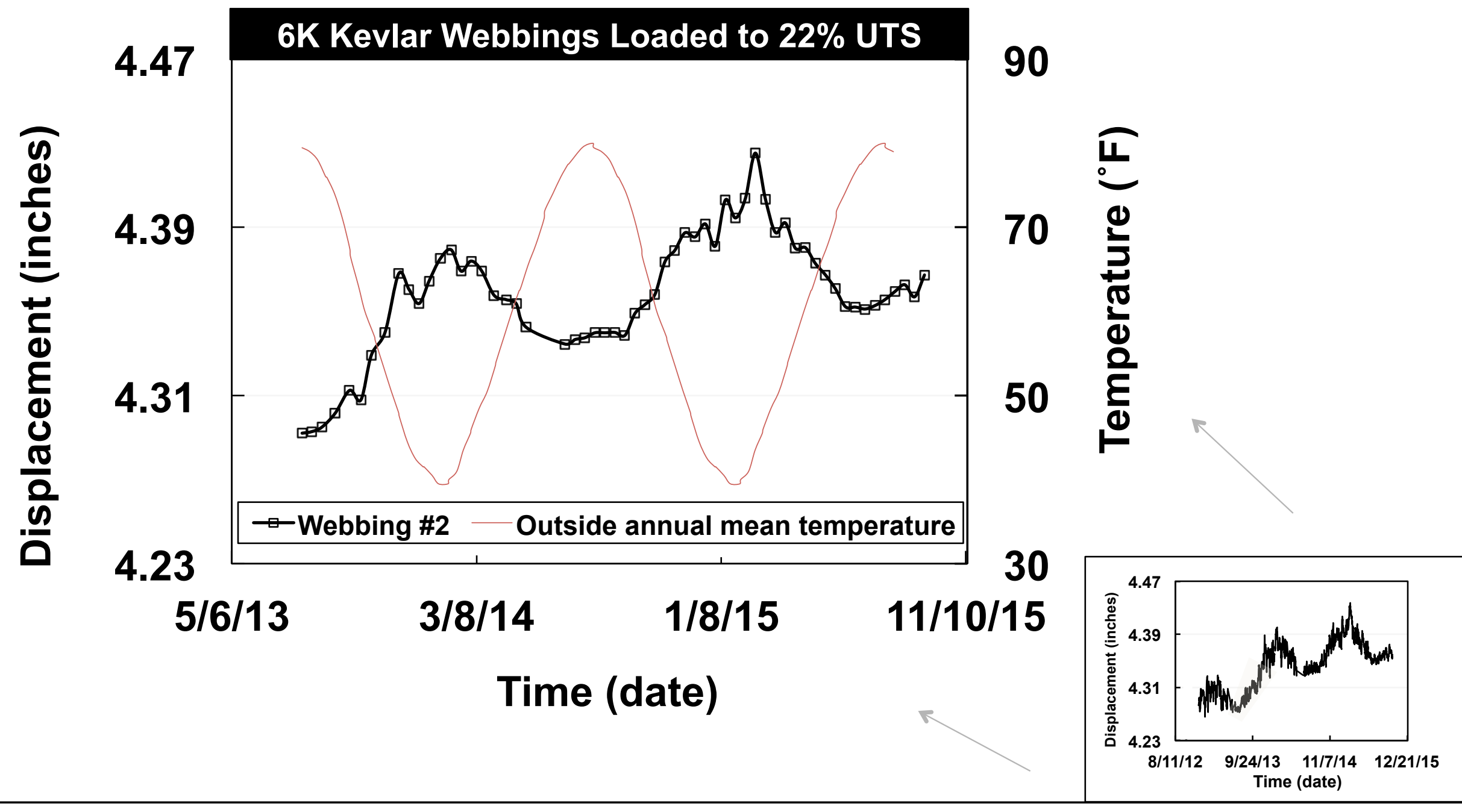

- Local wave patterns can be eliminated and global wave patterns highlighted through the use of running sum data

- Each data point in presented graph consist of 20 days of data 


\section{Running Sum Calculation}

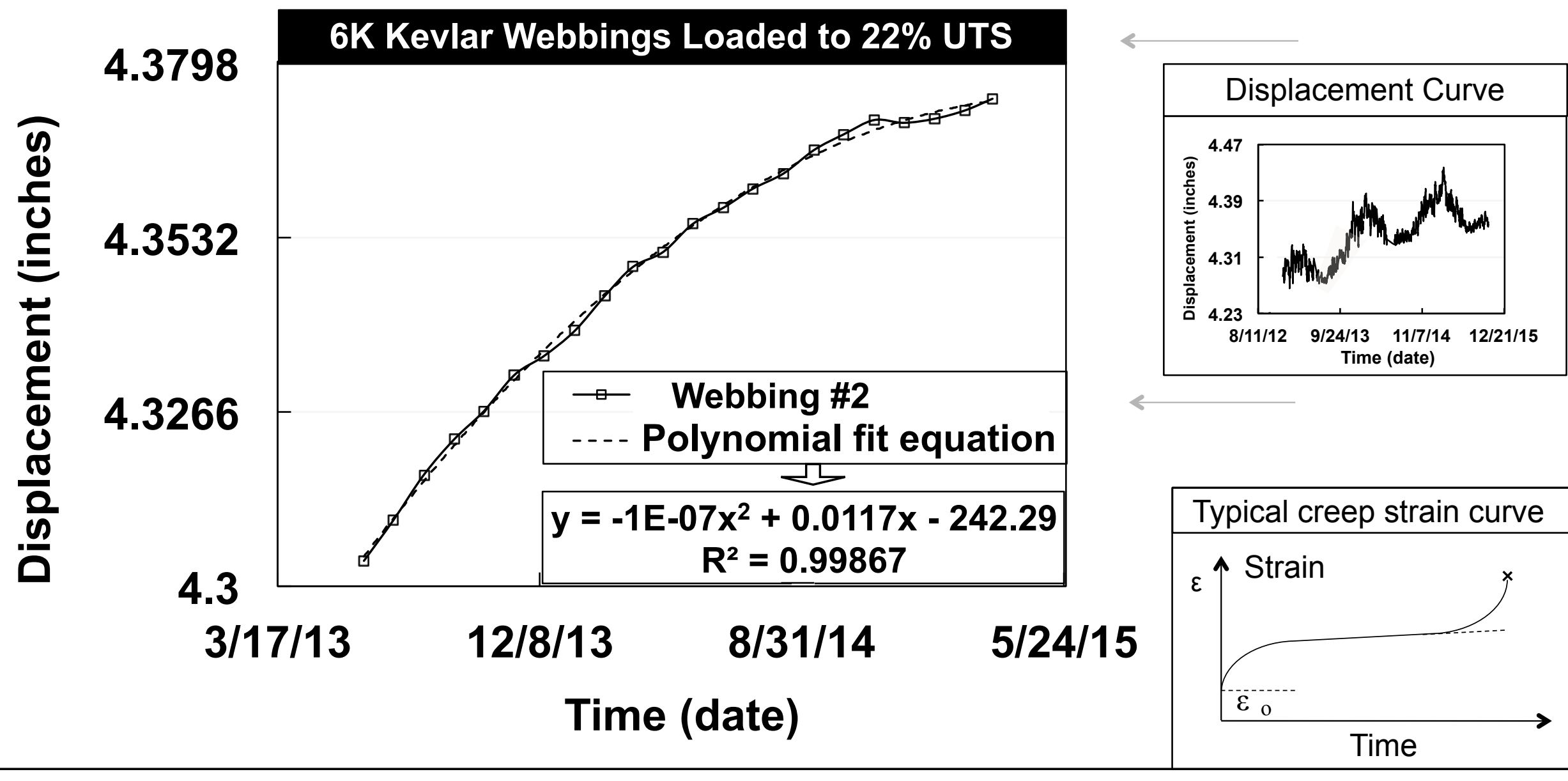

- All wave patterns can be eliminated and a continuous curve generated through the use of running sum calculations

- Each data point in presented graph consist of 1 year of data

- Running sum curve is comparable to a classic creep curve 
- Numerous Kevlar and Vectran webbings have maintained strength and supported a range of loads below $50 \%$ UTS over a four year period

- Cumulative visual and displacement data from the webbings indicate limited wear over the four year period - Damage defined by vertical incisions generate minute increases in displacement without detrimentally effecting long term strength

- Rotation has a detrimental effect on webbing strength - Utilization of running average calculations can smooth out short-term variations and highlight long-term trends 


\section{Future: Webbing Studies}

\section{Test Matrix}

\section{Environment Pristine (2012) $\quad$ Damaged (2014) $\quad$ Integrated Grid (2016)}

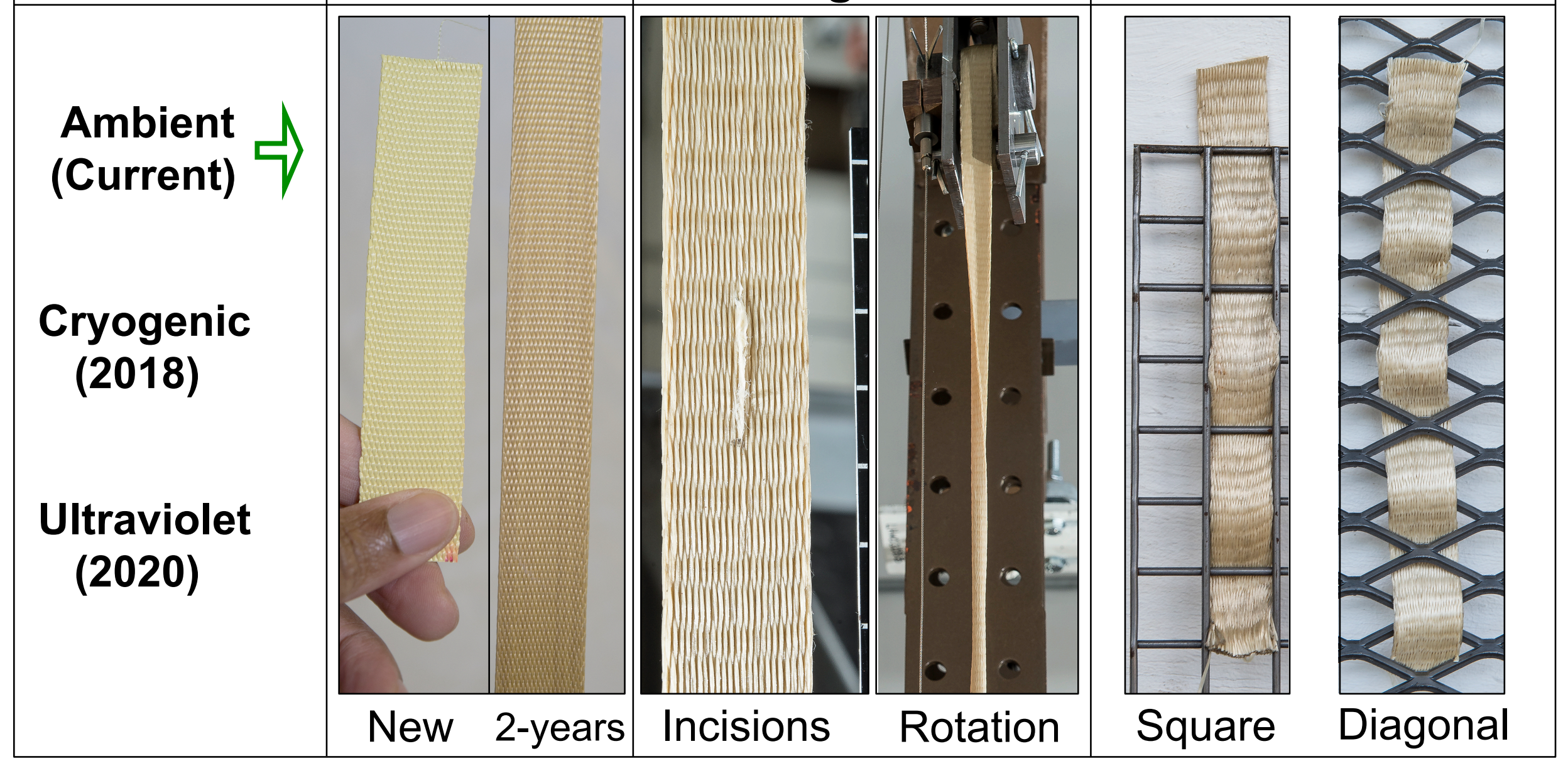

Goal: Experimentally validate long life and identify potential failure modes of webbings. 